\title{
Identification of immediate early response protein 2 as a regulator of angiogenesis through the modulation of endothelial cell motility and adhesion
}

\author{
WENJUAN WU ${ }^{1,2^{*}}$, XIZHI ZHANG ${ }^{2 *}$, HOUNING LV $^{1 *}$, YUEXIA LIAO $^{1 *}$, WEICHENG ZHANG ${ }^{1}$, \\ HAICHAO CHENG ${ }^{1}$, ZIJING DENG ${ }^{1}$, JINGYUAN SHEN ${ }^{1}$, QING YUAN ${ }^{1}$, YU ZHANG ${ }^{1}$ and WEIGAN SHEN ${ }^{1,3,4}$ \\ ${ }^{1}$ School of Medicine, ${ }^{2}$ Clinical Medical College, Yangzhou University, Yangzhou, Jiangsu 225001; \\ ${ }^{3}$ Jiangsu Key Laboratory of Integrated Traditional Chinese and Western Medicine for Prevention and Treatment \\ of Senile Diseases, Yangzhou, Jiangsu 225001; ${ }^{4}$ Jiangsu Co-innovation Center for Prevention and Control \\ of Important Animal Infectious Diseases and Zoonoses, Yangzhou, Jiangsu 225009, P.R. China
}

Received December 6, 2014; Accepted August 7, 2015

DOI: $10.3892 /$ ijmm.2015.2310

\begin{abstract}
Human immediate early response 2 (IER2) has been characterized as a putative nuclear protein that functions as a transcription factor or transcriptional co-activator in the regulation of cellular responses, and may be involved in the regulation of tumor progression and metastasis. Data from our previous gene expression profile of the human microvascular endothelial cells during capillary morphogenesis showed a significant alteration of IER 2 expression, suggesting that IER2 may participate in the regulation of the endothelial cell morphogenesis and angiogenesis. The aim of the present study was to investigate the role of IER2 in cell motility, cell-matrix adhesion and in vitro capillary-like structures formation of the human umbilical vein endothelium cells (HUVECs). IER2 was constitutively expressed in HUVECs, and lentiviral-mediated depletion of IER2 significantly reduced the cell motility, cell-matrix adhesion and capillary-like structures formation of HUVECs. Results also showed that depletion and overexpression of IER 2 altered the actin cytoskeleton rearrangement in HUVECs. Furthermore, results from western blot analysis showed that the activity of the focal adhesion kinase (FAK) can be regulated by IER2. These results indicated that IER2 regulates endothelial cell motility, adhesion on collagen type I matrix and the capillary tube formation, as the result of the regulation of the actin cytoskeleton rearrangement presumably via a FAK-dependent mechanism.
\end{abstract}

Correspondence to: Professor Weigan Shen, School of Medicine, Yangzhou University, 11 Huaihai Road, Yangzhou, Jiangsu 225001, P.R. China

E-mail: shenweigan@hotmail.com

*Contributed equally

Key words: human immediate early response 2, HUVECs, cell motility, cell-matrix adhesion, capillary-like structures formation

\section{Introduction}

Angiogenesis, the process of new vessels forming from preexisting microvasculature, is essential for tumor growth and metastasis, and is controlled by the balance between proangiogenic factors and angiogenesis inhibitors (1-4). Identifying the proangiogenic factors and inhibitors, and characterizing their contribution to endothelial cell motility and adhesion will aid the understanding of the angiogenesis process mechanism, but also provide potential candidates for developing numerous possible antiangiogenic therapeutic strategies targets for cancer.

Human immediate early response 2 (IER2, also known as Chxl or ETR101, a homologous gene in rat and mouse for pip92), a member of the immediate early responsible genes, was initially identified and cloned from the human myeloid leukemia cell line HL-60 (5), and found to be rapidly or transiently upregulated by extracellular stimuli, such as growth factors, cytokines, 12- $O$-tetradecanoylphorbol-13-acetate and certain pathogen infections (5-11). Human IER2 consists of 223 amino acids and is rich in proline, serine and arginine, and $76 \%$ of the amino acid residues encompassing the whole region are identical between human IER2 and mouse pip92. IER2 contains two putative nuclear localization signals and multiple phosphorylation sites, suggesting that IER2 may be a nuclear protein and require post-translational modifications. Currently, IER2 has been characterized as a putative nuclear protein that served function as a fibroblast growth factor intracellular binding protein 1-interacting partner (12), and as a transcription factor or transcriptional co-activator for the human myo-inositol 1-phosphate synthase gene involved in the regulation of cellular responses $(9,12)$. Furthermore, data from a previous study indicated that IER2 is involved in the regulation of tumor progression and metastasis (13), although the precise role and signaling mechanism involved remain elusive. In our previous study, microarray was performed to analyze the gene expression profile in response to basic fibroblast growth factor (bFGF), which is a proangiogenic factor (14), at selective time points during different phases of human dermal microvascular endothelial cells morphogenesis (15), and stimulation of HMVECs growing 
in fibrin matrices by bFGF led to a significant upregulation of IER 2 within $24 \mathrm{~h}$ and a subsequently significant downregulation between 24 and $72 \mathrm{~h}$ (data not shown), which prompted the evaluation of whether IER2 participates in the regulation of the endothelial cell morphogenesis and angiogenesis.

The aim of the present study was to investigate the role of IER2 in human endothelial cell motility, cell-matrix adhesion and in vitro capillary-like structures formation. To the best of our knowledge, this is the first demonstration that IER2 may be required for endothelial cell morphogenesis and in vitro angiogenesis.

\section{Materials and methods}

Cell lines and culture conditions. Human umbilical vein endothelium cells (HUVECs) were purchased from AllCells (Shanghai, China) and cultured in $0.5 \%$ gelatin-coated dishes in endothelial cell growth medium (EGM; AllCells) according to the manufacturer's instruction, and the passage numbers of the HUVECs were maintained between passage 3 and 8 . Human embryonic kidney (HEK) 293T cells, obtained from the Type Culture Collection of the Chinese Academy of Sciences (Shanghai, China), were grown in Dulbecco's modified Eagle's medium supplemented with $10 \%$ fetal bovine serum, $100 \mathrm{U} / \mathrm{ml}$ penicillin and $100 \mu \mathrm{g} / \mathrm{ml}$ streptomycin in a humidified incubator at $37^{\circ} \mathrm{C}$ with $5 \% \mathrm{CO}_{2}$.

Plasmid construction and lentivirus production. Recombinant lentiviruses encoding IER2 (LV-IER2) or control lentivirus (LV-CTL) were generated in HEK293T cells by co-transfecting the pEZ-Lv105-IER2 vector or pEZ-Lv105-null with the Lenti-Pac $^{\text {TM }}$ HIV Expression Packaging kit obtained from GeneCopoeia, Inc. (Rockville, MD, USA) according to the manufacturer's instruction. For lentiviral shRNA vector construction, three different shRNA corresponding to human IER2 (GenBank accession: NM_004907) were designed as follows: shR-1 forward, 5'-CCGGGACACAATCAGCCGAG AAGTTCTCGAGAACTTCTCGGCTGATTGTGTCTTT TTG-3' and reverse, 5'-AATTCAAAAAGACACAATCA GCCGAGAAGTTCTCGAGAACTTCTCGGCTGATTGT GTC-3'; shR-2 forward, 5'-CCGGCTACTTTCACATTC TCAAGTTCTCGAGAACTTGAGAATGTGAAAGTAGT TTTTG-3' and reverse, 5'-AATTCAAAA ACTACTTTC ACATTCTCAAGTTCTCGAGAACTTGAGAATGTGAA AGTAG-3'; and shR-3 forward, 5'-CCGGGATCTACTTT CACATTCTCAACTCGAGTTGAGAATGTGAAAGTAGA TCTTTTT G-3' and reverse, 5'-AATTCAAAAAGATCTA CTTTCACATTCTCAACTCGAGTTGAGAATGTGAAA GTAGATC-3'. The underlined sections refer to sequences corresponding to human IER2 (GenBank accession no. NM_004907) shRNA targets. The oligonucleotides were synthesized, annealed and cloned into as cloned into the lentiviral expression vector pGV115 obtained from Shanghai Genechem Co., Ltd., (Shanghai, China) at the AgeI and EcoRI sites. Recombinant lentiviruses (LV-shR-1, LV-shR-2 and LV-shR-3) were produced in HEK293T cells by co-transfection of the lentiviral shRNA vectors with the packaging vectors pHelper 1.0 and pHelper 2.0 purchased from Shanghai Genechem using Lipofectamine 2000 (Invitrogen, Carlsbad, CA, USA) according to the manufacturer's instruction. The lentiviruses were collected, purified and concentrated, and the infectious titers were determined by Shanghai Genechem. Non-silencing control shRNA lentivirus (LV-shCTL) was also obtained from Shanghai Genechem, and performed as the empty vectors for knockdown gene expression. For lentiviral transduction, HUVECs were seeded in the $0.5 \%$ gelatin-coated 6 -well culture plates, and were subsequently infected with the indicated lentiviruses at a multiplicity of infection of 30 in the presence of polybrene $(5 \mu \mathrm{g} / \mathrm{ml})$, and cultured for $>96 \mathrm{~h}$ in EGM. The green fluorescence protein, co-expressed in the lentivirus-transduced cells, served as a selection marker to indicate the successfully transduced HUVECs.

$R N A$ extraction and reverse transcription-quantitative polymerase chain reaction ( $R T-q P C R)$. Total RNA was extracted from HUVECs using the RNA isolator Total RNA extraction reagent (Vazyme Biotech Co., Ltd., Nanjing, China), according to the manufacturer's instruction, and reverse transcribed using the HiScript First Strand cDNA Synthesis kit (Vazyme Biotech). RT-qPCR was performed on an ABI 7500 real-time PCR system (Applied Biosystems, Foster City, CA, USA) using the AceQ ${ }^{\circledR}$ qPCR SYBR ${ }^{\circledR}$ Green Master mix kit (Vazyme Biotech Co., Ltd.) according to the manufacturer's instructions. To discriminate between endogenous versus exogenous IER2, the following were designed: Primers I sense, 5'-TGG TGAAACTGGGCCAATCT-3' and antisense, 5'-AAGAAT CCACCGCACGAAAG-3', which spanned the 3'-untranslated region (UTR) of the IER2 gene and only recognized the endogenous mRNA of IER2, and primers II sense, 5'-CCA AAGTCAGCCGCAAACGA-3' and antisense, 5'-TTTCTT CCAGACGGGCTTTCTTGC-3', which spanned the coding region and recognized the endogenous and exogenous IER2 gene. Primers for GAPDH were sense, 5'-GCACCGTCAAGG CTGAGAAC-3' and antisense, 5'-TGGTGAAGACGCCAG TGGA-3'. The expression of the IER 2 mRNA was normalized to GAPDH mRNA, and the fold changes in the expression of IER 2 were evaluated by the $2^{-\triangle \Delta \mathrm{CT}}$ method.

Western blot analysis. HUVECs were washed with ice-cold phosphate-buffered saline (PBS) and lysed with a total protein extraction kit including Protease Inhibitor mix (Vazyme Biotech Co., Ltd.). Protein concentrations were determined using the Bradford protein quantification kit (Vazyme Biotech Co., Ltd.). Total protein was separated by SDS-polyacrylamide gel electrophoresis (SDS-PAGE), and transferred to polyvinylidene fluoride membranes (Millipore, Billerica, MA, USA). Following blocking in PBS-Tween-20 containing 5\% non-fat dried milk, the membranes were incubated at $4^{\circ} \mathrm{C}$ overnight with mouse polyclonal anti-IER2 (Cat. no. ab168980; 1:1,000; Abcam, Cambridge, MA, USA), rabbit monoclonal anti-focal adhesion kinase (FAK; Cat. no. 13009), rabbit polyclonal anti-pY397FAK (Cat. no. 3283; 1:1,000; Cell Signaling Technology, Danvers, MA, USA) or mouse monoclonal anti-GAPDH (Cat. no. KC-5G4; 1:1,000; KangChen Bio-tech, Inc., Shanghai, China) primary antibodies, followed by incubation for $2 \mathrm{~h}$ at room temperature with horseradish peroxidase-conjugated goat anti-mouse immunoglobulin G (IgG; Cat. no. KC-MM-035) or goat anti-rabbit IgG (Cat. no. KC-RB-035) antibodies (1:2,000; KangChen Bio-tech, Inc.), and visualized using the Pierce ECL Plus Western Blotting Substrate kit (Thermo Fisher Scientific, Rockford, IL, USA). 
Cell viability analysis. Cell viability assay was performed using the 3-(4,5-dimethylthiazol-2-yl)-2,5-diphenylterazolium bromide (MTT) and the Cell Counting kit-8 (CCK-8; Obio Technology, Shanghai, China) assays. For the MTT assay, $100 \mu \mathrm{l}$ of HUVECs $\left(3 \times 10^{4}\right.$ cells/well) suspended in EGM were plated in the 96 -well culture plates pre-coated with $0.5 \%$ gelatin. After incubation at $37^{\circ} \mathrm{C}$ for $24 \mathrm{~h}, 20 \mu \mathrm{l}$ of MTT solution $(5 \mathrm{mg} / \mathrm{ml}$; Sigma St. Louis, MO, USA) was added to each well, and the samples were incubated for another $4 \mathrm{~h}$ at $37^{\circ} \mathrm{C}$. Subsequently, the MTT solution was removed and $100 \mu$ l of dimethyl sulfoxide was added to dissolve the formazan crystals followed by absorbance measurement at $570 \mathrm{~nm}$ using a multifunctional microplate reader (BioTek Instruments, Winooski, VT, USA). The CCK-8 assay was also performed according to the manufacturer's instructions. After incubation at $37^{\circ} \mathrm{C}$ for $24 \mathrm{~h}, 10 \mu \mathrm{l}$ of CCK-8 solution was added to each well and incubated for another $1 \mathrm{~h}$ at $37^{\circ} \mathrm{C}$, and the absorbance at $450 \mathrm{~nm}$ was measured.

Cell migration and invasion analysis. The Transwell migration assay was used to evaluate the capacity of HUVEC migration. In brief, HUVECs were serum-starved overnight and $200 \mu \mathrm{l}$ of $1 \times 10^{5}$ cells were seeded in the upper chamber (with $8.0-\mu \mathrm{m}$ pore size; Costar, Corning, NY, USA) of the 24-well Transwell, which was subsequently placed into the lower chamber containing $600 \mu \mathrm{l}$ of EGM, and allowed to migrate toward the underside of the insert for $24 \mathrm{~h}$ at $37^{\circ} \mathrm{C}$. Cells on the upper surface of the inserts were gently removed, and the cells on the lower surface of the filter were fixed with methanol and stained with $1 \%$ crystal violet. The number of migrated cells in each chamber was counted in $\geq 5$ randomly selected fields (magnification, $x 200$ ), and images were captured under a light phase contrast microscopy equipped with a digital image capturing system. The cell invasion assay was also performed in the Transwell chambers with the minor modification that the transwell inserts were pre-coated with $100 \mu \mathrm{g} / \mathrm{ml}$ Matrigel (BD Biosciences, San Jose, CA, USA), and $2 \times 10^{5}$ cells were plated into the upper chambers.

Cell-matrix adhesion analysis. The cell-matrix adhesion assay was performed in 96-well culture plates pre-coated with $10 \mu \mathrm{g} / \mathrm{ml}$ collagen type I (BD Biosciences) overnight at $4^{\circ} \mathrm{C}$. After blocking with $1 \%$ heat-denatured bovine serum albumin in PBS at $37^{\circ} \mathrm{C}$ for $\geq 1 \mathrm{~h}$, HUVECs $\left(2 \times 10^{5}\right.$ cells/well $)$ were added to each well and incubated at $37^{\circ} \mathrm{C}$ for $1 \mathrm{~h}$. Unbound cells were gently removed and attached cells were quantified by the CCK- 8 assay.

In vitro capillary-like tube formation analysis. To test the angiogenic activity, the capillary-like tube formation by HUVECs on Matrigel was performed in vitro. In brief, a pre-chilled 96-well culture plate was coated with $50 \mu$ l of Matrigel, which was previously thawed at $4^{\circ} \mathrm{C}$ overnight, and allowed to polymerize for $\geq 1 \mathrm{~h}$ at $37^{\circ} \mathrm{C}$. HUVECs $\left(3 \times 10^{4}\right.$ cells/well) were plated in coated wells and incubated at $37^{\circ} \mathrm{C}$ for $24 \mathrm{~h}$. The tube formation was observed using an inverted phase contrast microscope and analyzed with the ImageJ software (http://imagej.nih.gov/ij/). Only the complete ring structures created by HUVECs were counted as tubes.

Immunofluorescence analysis. HUVECs were seeded in coverslips coated with $0.5 \%$ gelatin in 24 -well plates and
A

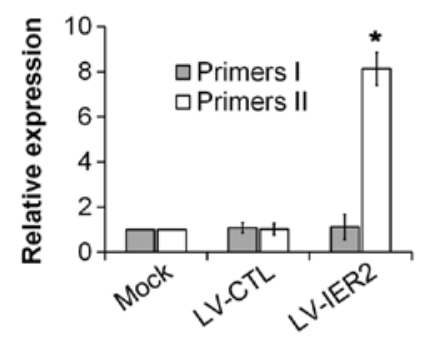

C
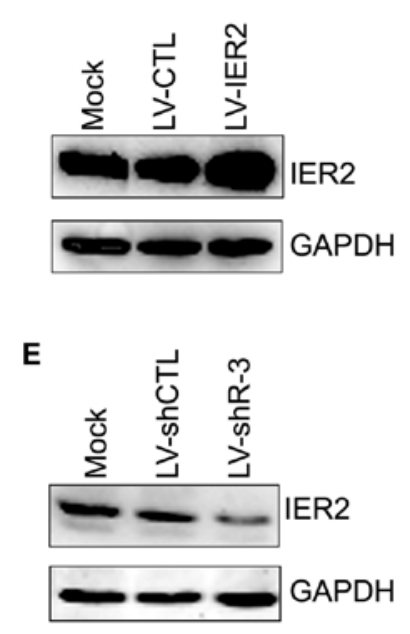

B

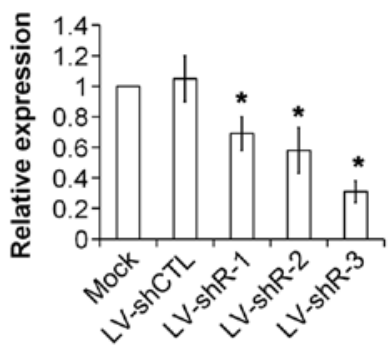

D

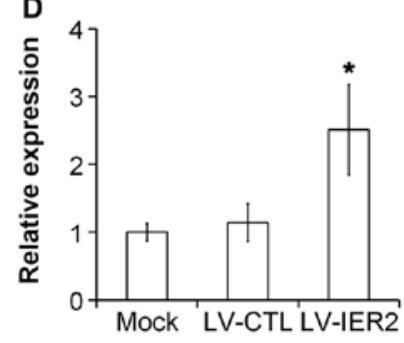

$\mathbf{F}$

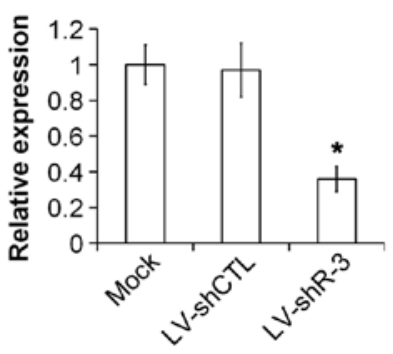

Figure 1. Expression of IER2 in HUVECs. (A and B) IER2 mRNA levels were quantified in RNA extracts from the indicated lentivirus-transduced HUVECs using reverse transcription-quantitative polymerase chain reaction normalized to GAPDH. The values represent the mean $\pm \mathrm{SD}(\mathrm{n}=5)$. ${ }^{*} \mathrm{P}<0.05$ vs. the mock. (C-F) Overexpression and knockdown of IER2 were determined by SDS-PAGE immunoblotting with antibodies to IER2 and GAPDH, normalized to GAPDH. The values represent the mean \pm SD $(n=3)$. ${ }^{*} \mathrm{P}<0.05$, vs. the mock. IER2, human immediate early response 2; HUVECs, human umbilical vein endothelium cells; SD, standard deviation; LV, lentivirus.

incubated in EGM at $37^{\circ} \mathrm{C}$ for $24 \mathrm{~h}$, and subsequently were fixed with $4 \%$ paraformaldehyde for $30 \mathrm{~min}$, permeabilized with $0.5 \%$ Triton X-100 for $10 \mathrm{~min}$, and blocked with Image-iT FX Signal Enhancer (Invitrogen) for $30 \mathrm{~min}$. Afterwards cells were washed twice with PBS and subsequently incubated with the rhodamine-conjugated phalloidin (Sigma) for $1 \mathrm{~h}$ at room temperature. The coverslips were washed thoroughly with PBS, mounted on glass slides and analyzed under a fluorescent microscope equipped with a digital image capturing system.

Statistical analysis. Data are expressed as mean \pm standard deviation. Statistical significance was determined by the Student's t-test, and $\mathrm{P}<0.05$ was considered to indicate a statistically significant difference.

\section{Results}

IER2 expression in HUVECs. Initially, the expression of IER2 in HUVECs after indicated lentiviruses transduction was examined. As shown in Fig. 1, the levels of endogenous and the total (endogenous plus exogenous) IER $2 \mathrm{mRNA}$ was measured by 

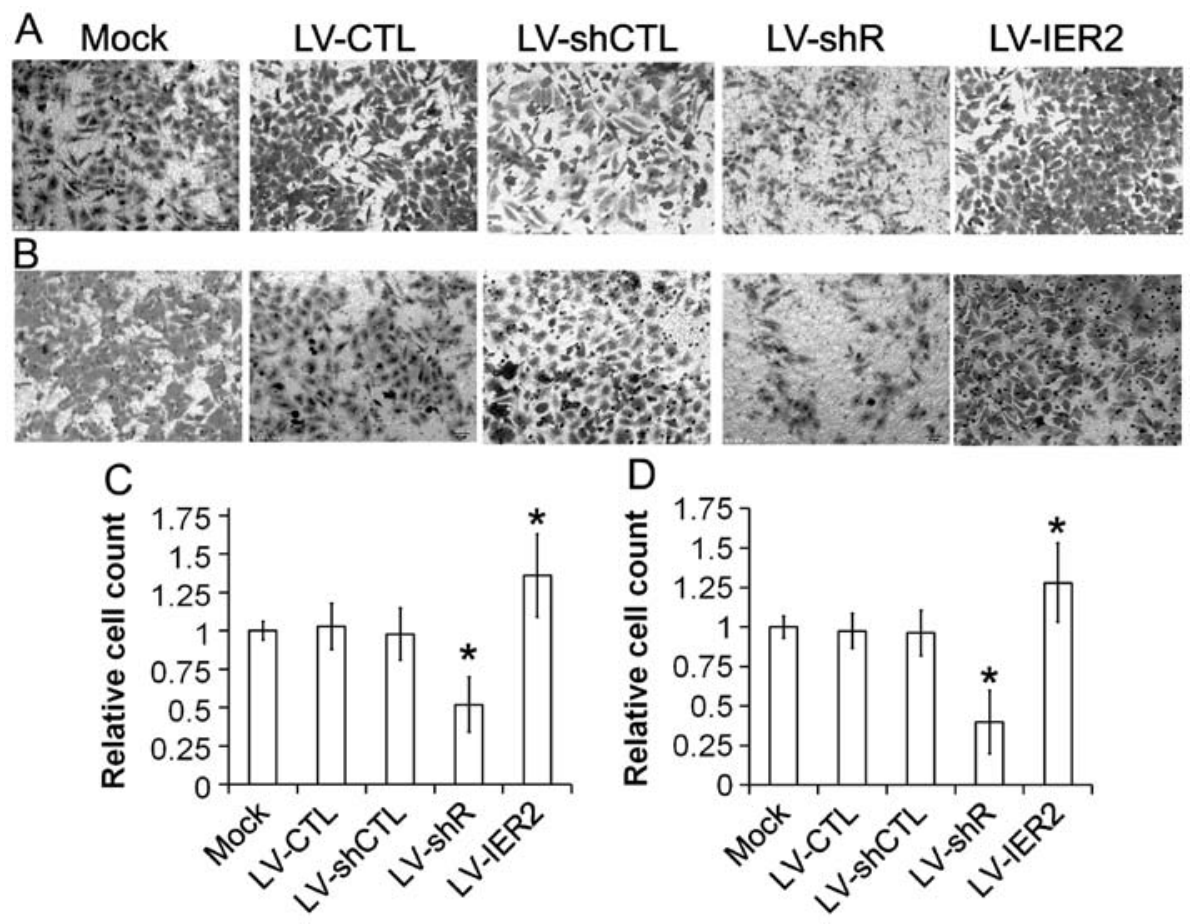

Figure 2. Effects of human immediate early response 2 on HUVEC migration and invasion. The lentivirus-transduced HUVECs were plated in the Transwell inserts (A) uncoated or (B) precoated with Matrigel, respectively, and cell migration or invasion towards the low chambers were recorded from $\geq 5$ randomly selected fields in each chamber from triplicate wells (magnification, x200). Quantification of the (C) migration and (D) invasion of the lentivirus-transduced HUVECs are shown. Assays were performed in triplicate for each experiment, and repeated three times. The values represent the mean \pm standard deviation. ${ }^{*} \mathrm{P}<0.05$, vs. the mock. HUVECs, human umbilical vein endothelium cells; LV, lentivirus.

RT-qPCR using primers I and II, respectively. Overexpression of IER2 mRNA (total IER2 mRNA) was observed in LV-IER2-transduced HUVECs, and the levels of endogenous IER2 mRNA were induced, but not significantly (Fig. 1A), suggesting that LV-IER2 infection did not affect the expression of endogenous IER2. However, the knockdown of IER2 expression by LV-shR-3 was achieved with the most potent inhibition at the mRNA levels (Fig. 1B). Data from the western blot assay showed that IER2 was constitutively expressed in the transduced empty vectors (LV-CTL and LV-shCTL), and the untransduced control HUVECs (mock) (Fig. 1C and E), and upregulation and efficient inhibition of the IER2 protein were shown in LV-IER2and LV-shR-3-transduced HUVECs, respectively (Fig. 1C-F). These data indicated that lentiviruses were successfully transduced into HUVECs, and that IER2 was overexpressed in LV-IER2-transduced HUVECs, and the most efficient inhibition of IER2 was shown in LV-shR-3-transduced cells.

IER2 regulates endothelial cell migration and invasion. Results from the Transwell cell migration analysis showed that depletion of IER2 in HUVECs by infection with LV-shR significantly reduced the number of the motile cells, while overexpression of IER 2 by LV-IER 2 infection significantly promoted the HUVECs migration (Fig. 2A and C) in comparison with empty vector-transduced cells and the mock. Similar effects of IER2 knockdown and overexpression on the HUVECs invasion were also shown in the Transwell cell invasion analysis (Fig. 2B and D). Furthermore, the alteration in cell motility induced by knockdown and overexpression of IER2 did not appear to be due to decreased cell viability. As shown in Fig. 3, knockdown and overexpression of IER2 had a
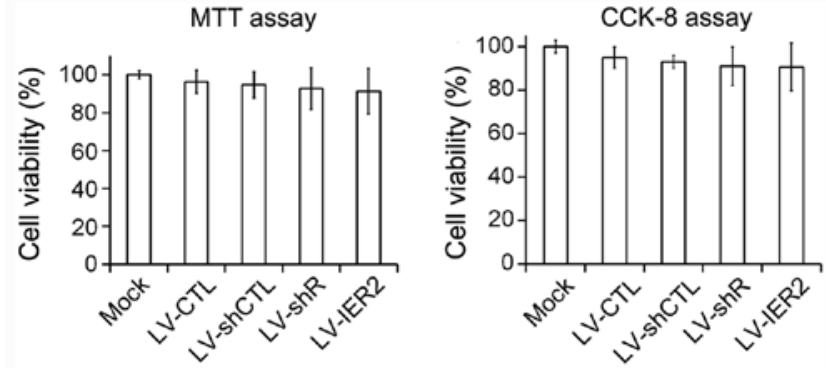

Figure 3. Effect of human immediate early response 2 on the cell viability. The lentivirus-transduced human umbilical vein endothelium cells were plated in the 96-well culture plates. Cell viability was measured using the MTT and CCK-8 assays by absorbance at 570 and $450 \mathrm{~nm}$, respectively. Assays were performed in 6-wells and repeated five times. The data are expressed as the percentages of the mock, and the values represent the mean \pm standard deviation. LV, lentivirus; CCK-8, cell counting kit-8.

non-significant decrease on the cell viability compared to that of empty vector-transduced cells and the mock. These results demonstrated that depletion and overexpression of IER2 modulated the general capacity of endothelial cell motility, and the endogenous IER2 expression may be required for endothelial cell migration and invasion.

IER2 regulates HUVECs adhesion onto the collagen type I matrix. Endothelial cell adhesion onto the extracellular matrix has a central role in endothelial cell migration and angiogenesis $(16,17)$. To examine the role of IER2 in the cell-matrix adhesion, HUVECs were seeded in collagen type I-coated 96-well plates and incubated at $37^{\circ} \mathrm{C}$ for $1 \mathrm{~h}$. As shown in Fig. 4, LV-shR infection caused a significant reduction of 


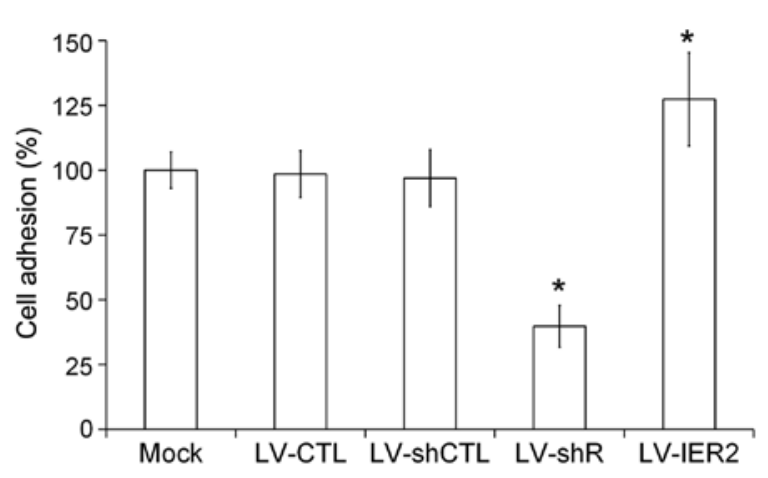

Figure 4. Effect of human immediate early response 2 on HUVECs-matrix adhesion. The lentivirus-transduced HUVECs were seeded in the 96-well culture plate pre-coated with collagen type I and incubated at $37^{\circ} \mathrm{C}$ for $1 \mathrm{~h}$. The number of the adhesive cells was quantified using the cell counting kit- 8 assay by absorbance at $450 \mathrm{~nm}$. Assays were performed in 6 -wells and repeated five times. The reported values are expressed as the percentages of the mock, and represent the mean \pm standard deviation. ${ }^{*} \mathrm{P}<0.05$, vs. the mock. HUVECs, human umbilical vein endothelium cells; LV, lentivirus.

HUVECs adhesion onto collagen type I, while LV-IER2 infection promoted HUVECs cell adhesion onto the matrix compared to those in the empty vector-transduced cells and the mock, suggesting that IER2 may be an important regulator of endothelial cell adhesion to the matrix, and the endogenous IER 2 expression may be required for endothelial cell adhesion to the matrix and motility.

IER2 regulates the capillary tube formation in vitro of HUVECs via a FAK-dependent mechanism. Endothelial cells plated on the extracellular matrix may rapidly attach and form capillary-like tube structures (18). To examine the role of IER2 in endothelial cell differentiation into capillary-like structures, the in vitro tube formation assay was performed. As indicated in Fig. 5A and B, a spontaneous differentiation of HUVECs into capillary-like tubes was identified in the mock, empty vector- and LV-IER2-transduced HUVECs, particularly in LV-IER2-transduced cells, while knockdown of IER2 clearly disrupted the HUVECs capillary morphogenesis, suggesting that IER2 may act as a regulator in HUVECs differentiation into capillary-like structures in vitro. As endothelial FAK expression and activity have been implicated as the important modulators of angiogenesis $(4,16,19)$, the western blot assay was subsequently performed to evaluate the effects of IER2 on the FAK expression, the phosphorylation of which is known to regulate cell-matrix adhesion during cell migration. As shown in Fig. 5C and D, knockdown of IER2 in HUVECs by LV-shR infection clearly downregulated the phosphorylation level of FAK at Y397 (pY397FAK), while upregulation of pY397FAK was shown in LV-IER2-transduced HUVECs, and no significant effects on the level of total FAK were observed in HUVECs with either knockdown or overexpression of IER2. LV-CTL or LV-shCTL transduction in HUVECs did not cause a significant alteration of pY397FAK and total FAK (data not shown). Taken together, the data suggested that IER2 may regulate the endothelial cell-matrix adhesion, cell migration and invasion during in vitro capillary tube formation of HUVECs, possibly via a FAK involved manner. Studies of the effects of IER2 on the regulation of the FAK-mediated signal pathway during angiogenesis are under way.

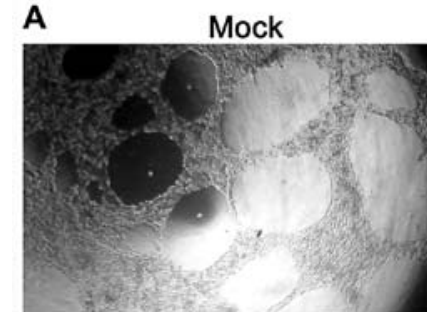

LV-shCTL

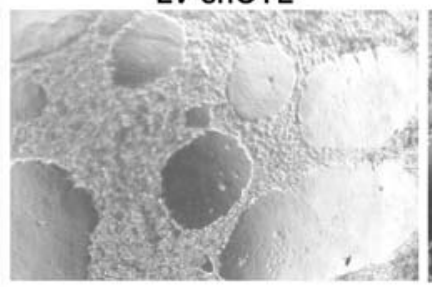

LV-IER2
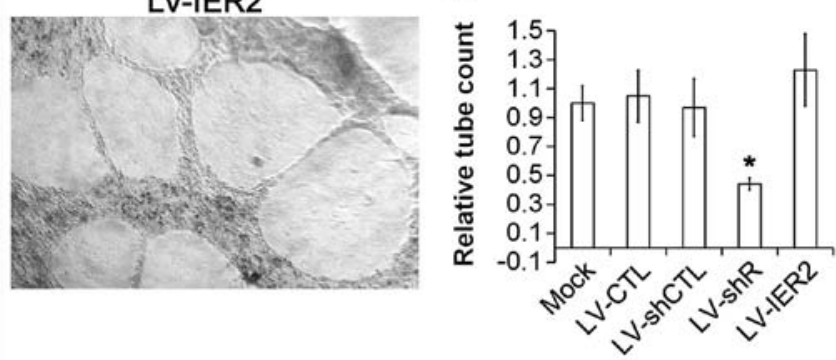

C

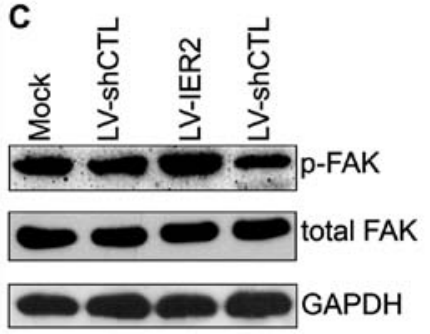

D

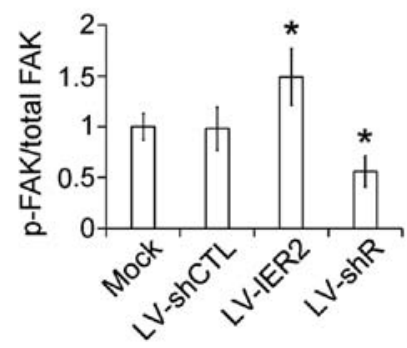

Figure 5. Effect of IER2 on HUVECs capillary-like tubules formation in vitro and FAK expression. (A) Representative images of preformed capillary-like tubules in the indicated lentivirus-transduced HUVECs are shown (magnification, x100) from three random selected fields in each well from triplicate experiments. (B) Quantitative analysis of the effects of IER2 on the capillarylike tubules formation of HUVECs. The values represent the mean \pm SD from three independent experiments. ${ }^{*} \mathrm{P}<0.05$, vs. the mock. (C) HUVECs were harvested and lysed for the detection of FAK and pY397FAK expression by western blot analysis. GAPDH was used as loading control. (D) Quantification of the band intensity was performed with ImageJ software, and expressed as folds of the mock subsequent to normalizing with GAPDH. Data represent the mean \pm SD of three independent experiments. "P<0.05, vs. the mock. IER2, human immediate early response 2 ; HUVECs, human umbilical vein endothelium cells; SD, standard deviation; LV, lentivirus.

IER2 regulates the actin cytoskeleton rearrangement. Considering endothelial cell migration, adhesion and capillary morphogenesis require dynamic remodeling of the actin microfilaments, whether IER2 could regulate the actin cytoskeleton arrangement in HUVECs was further examined by staining with the rhodamine-conjugated phalloidin. As shown in Fig. 6, the formation of the filopodia and lamellipodia at the leading edge were shown in the mock, empty vector- and LV-IER2-transduced HUVECs, particularly in LV-IER2-transduced cells, while IER2 depletion clearly disrupted or inhibited the formation of the filopodia and 


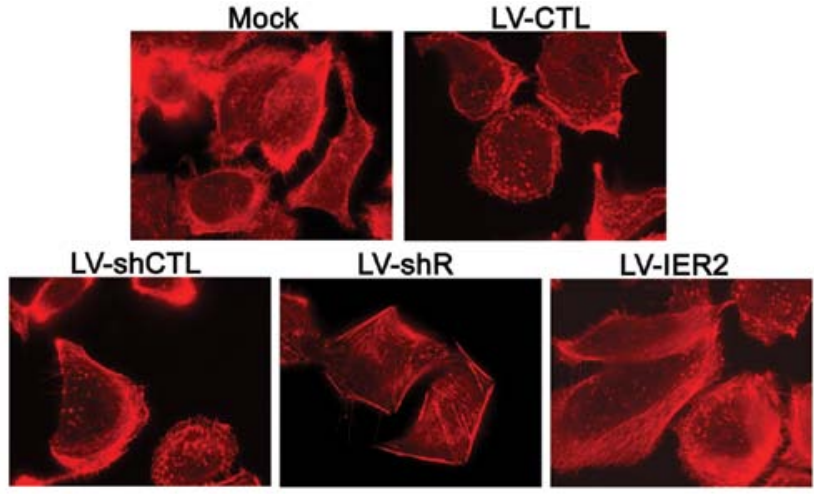

Figure 6. Effect of human immediate early response 2 on the actin microfilaments in HUVECs. HUVECs were fixed, permeabilized, blocked with Image-iT FX Signal Enhancer, and stained with rhodamine-conjugated phalloidin. Scale bars, $20 \mu \mathrm{m}$. The images represent the cells from three independent experiments. HUVECs, human umbilical vein endothelium cells.

lamellipodia at the leading edge of the HUVECs. These data suggested that the altered cell motility, cell-matrix adhesion and the capillary tube formation of HUVECs caused by IER2 depletion and overexpression may be the consequence of the regulation of the actin cytoskeleton rearrangement.

\section{Discussion}

In the present study, the HUVEC cells were used to study the role of IER2 in cell motility, matrix adhesion and capillary-like tube formation in vitro, which are the key steps for angiogenesis, and provided the first evidence for a role of IER2 as a potential regulator for endothelial cell morphogenesis and in vitro angiogenesis.

Our previous study reported gene expression profile analysis of the human microvascular endothelial cells during capillary morphogenesis (15), and identified a significant alteration of IER2 expression at selective time-points (data not shown), suggesting that IER2 may be involved in the regulation of the endothelial cell morphogenesis and angiogenesis. As endothelial cell motility has an important role in the maintenance of vascular function (20), whether IER2 regulates endothelial cell migration and invasion was initially evaluated. The present study demonstrated that IER 2 was constitutively expressed in HUVECs, and overexpression of IER 2 promoted HUVECs migration and invasion as shown by the Transwell cell migration and invasion assays, respectively. To determine whether the increased cell motility is due to the exogenous IER2 and not the endogenous IER2, depletion of IER2 in HUVECs was performed by infection with LV-shR, which directed against the 3'-UTR of the IER 2 mRNA and only knocked down the endogenous IER 2 . Knockdown of the endogenous IER 2 by LV-shR infection significantly decreased the HUVECs migration and invasion. This result was in accordance with another study showing that IER2 promoted cell motility and tumor metastasis (13). These findings suggested that knockdown and overexpression of IER2 modulated the general capacity of endothelial cell motility, and the endogenous IER2 expression may be required for endothelial cell migration and invasion.
The present study further demonstrated that silencing of IER2 expression resulted in a significant reduction of HUVECs adhesion onto the collagen type I substrate, while overexpression of IER2 increased the adhesive capacity of HUVECs to collagen type I. As endothelial cell-matrix adhesion is a principal requirement for cell migration during angiogenesis (21), these data clearly indicated that IER2 may be an important regulator of endothelial cell migration and adhesion to the substratum, which are critical features of endothelial cell differentiation into capillary tube-like structures and vessel formation. To test whether endogenous IER2 expression is required for endothelial cell differentiation into capillary-like structures, the in vitro tube formation assay was performed. Inhibition of IER2 expression in HUVECs significantly decreased the capillary tube-like structures formation. These findings provided the first evidence that IER2 may be a novel regulator in the modulation of angiogenic key events. During angiogenesis, endothelial cells sense the motile stimuli by filopodia, extend protrusions or lamellipodia at the leading edge, migrate and aggregate in the cell-matrix adhesion to form vessels structures $(16,22)$. In the present study, inhibition of IER 2 clearly disrupted or decreased the formation of the filopodia and lamellipodia at the leading edge of the HUVECs, which was in agreement to the decrease of cell motility, cell-matrix adhesion and in vitro tube-like structure formation, as mentioned above.

Considering that endothelial FAK expression and activity are essential for cell motility, cell-matrix adhesion and actin polymerization $(4,16,19)$, and the phosphorylation of FAK at Y397 is known to be the first step for the activation of the FAK-mediated signal pathway to regulate cell-matrix adhesion and migration $(23,24)$, the effects of IER2 on the FAK expression were evaluated. Depletion of IER2 expression significantly downregulated the level of pY397FAK in HUVECs, while overexpression upregulated the level of pY397FAK. Studies of the effects of IER2 expression on the regulation of other molecular levels and activity in the FAK-mediated signaling pathway during angiogenesis are under way. Taken together, these findings suggested that IER2 regulates endothelial cell motility, adhesion on collagen type I matrix and the capillary tube formation as the result of the regulation of the actin cytoskeleton rearrangement presumably via a FAK-dependent mechanism.

In conclusion, the present study demonstrated that IER2 is constitutively expressed in HUVECs, and is essential for endothelial cell motility, adhesion on collagen type I matrix and the capillary tube formation in vitro. IER 2 regulated endothelial cell motility, cell-matrix adhesion and the capillary tube formation of HUVECs as the result of modulation of the remodeling of actin cytoskeleton presumably through a FAK-dependent mechanism. Although further study is required to identify potential partners or targets of IER2 in regulating FAK activity, the results presented may provide a novel therapeutic target to inhibit angiogenesis.

\section{Acknowledgements}

The present study was supported by the National Nature Science Foundation of China (grant no. 81172278) and the Innovation and Entrepreneurship Training Program of Jiangsu College Students (grant no. 201311117060Y). 


\section{References}

1. Folkman J: Role of angiogenesis in tumor growth and metastasis. Semin Oncol 29 (Suppl 16): 15-18, 2002.

2. Birdsey GM, Dryden NH, Amsellem V, Gebhardt F, Sahnan K, Haskard DO, Dejana E, Mason JC and Randi AM: Transcription factor Erg regulates angiogenesis and endothelial apoptosis through VE-cadherin. Blood 111: 3498-3506, 2008.

3. Iruela-Arispe ML and Dvorak HF: Angiogenesis: A dynamic balance of stimulators and inhibitors. Thromb Haemost 78 672-677, 1997.

4. Cabrita MA, Jones LM, Quizi JL, Sabourin LA, McKay BC and Addison C: Focal adhesion kinase inhibitors are potent anti-angiogenic agents. Mol Oncol 5: 517-526, 2011.

5. Shimizu N, Ohta M, Fujiwara C, Sagara J, Mochizuki N, Oda $\mathrm{T}$ and Utiyama $\mathrm{H}$ : Expression of a novel immediate early gene during 12-O-tetradecanoylphorbol-13-acetate-induced macrophagic differentiation of HL-60 cells. J Biol Chem 266 12157-12161, 1991.

6. Deng YJ, Huang ZX, Zhou CJ, Wang JW, You Y, Song ZQ, Xiang MM, Zhong BY and Hao F: Gene profiling involved in immature $\mathrm{CD} 4^{+} \mathrm{T}$ lymphocyte responsible for systemic lupus erythematosus. Mol Immunol 43: 1497-1507, 2006.

7. Shen QY and Zheng SS: Identification of genes differentially expressed in monocyte-derived dendritic cells with 1á, 25-dihydroxyvitamin D3 using cDNA arrays. J Zhejiang Univ SCI 5: 222-225, 2004

8. Zeng F, Hon CC, Sit WH, Chow KY, Hui RK, Law IK, Ng VW, Yang XT, Leung FC and Wan JM: Molecular characterization of Coriolus versicolor PSP-induced apoptosis in human promyelotic leukemic HL-60 cells using cDNA microarray. Int J Oncol 27: 513-523, 2005

9. Takaya T, Kasatani K, Noguchi S and Nikawa J: Functional analyses of immediate early gene ETR 101 expressed in yeast. Biosci Biotechnol Biochem 73: 1653-1660, 2009.

10. Chen L, Ma S, Li B, Fink T, Zachar V, Takahashi M, Cuttichia J, Tsui LC, Ebbesen P and Liu X: Transcriptional activation of immediate-early gene ETR 101 by human T-cell leukaemia virus type I Tax. J Gen Virol 84: 3203-3214, 2003.

11. Hess S, Rheinheimer C, Tidow F, Bartling G, Kaps C, Lauber J, Buer J and Klos A: The reprogrammed host: Chlamydia trachomatis-induced up-regulation of glycoprotein 130 cytokines, transcription factors, and antiapoptotic genes. Arthritis Rheum 44: 2392-2401, 2001.

12. Hong SK and Dawid IB: FGF-dependent left-right asymmetry patterning in zebrafish is mediated by Ier2 and Fibp1. Proc Natl Acad Sci USA 106: 2230-2235, 2009.
13. Neeb A, Wallbaum S, Novac N, Dukovic-Schulze S, Scholl I, Schreiber C, Schlag P, Moll J, Stein U and Sleeman JP: The immediate early gene Ier 2 promotes tumor cell motility and metastasis, and predicts poor survival of colorectal cancer patients. Oncogene 31: 3796-3806, 2012

14. Bayless KJ and Davis GE: The Cdc42 and Rac1 GTPases are required for capillary lumen formation in three-dimensional extracellular matrices. J Cell Sci 115: 1123-1136, 2002.

15. Sun XT, Zhang MY, Shu C, Li Q, Yan XG, Cheng N, Qiu YD and Ding YT: Differential gene expression during capillary morphogenesis in a microcarrier-based three-dimensional in vitro model of angiogenesis with focus on chemokines and chemokine receptors. World J Gastroenterol 11: 2283-2290, 2005.

16. Kuo MW, Wang CH, Wu HC, Chang SJ and Chuang YJ: Soluble THSD7A is an N-glycoprotein that promotes endothelial cell migration and tube formation in angiogenesis. PLoS One 6: e29000, 2011.

17. Otrock ZK, Mahfouz RA, Makarem JA and Shamseddine AI: Understanding the biology of angiogenesis: Review of the most important molecular mechanisms. Blood Cells Mol Dis 39: 212-220, 2007.

18. Kubota Y, Kleinman HK, Martin GR and Lawley TJ: Role of laminin and basement membrane in the morphological differentiation of human endothelial cells into capillary-like structures. J Cell Biol 107: 1589-1598, 1988.

19. Braren R, Hu H, Kim YH, Beggs HE, Reichardt LF and Wang R: Endothelial FAK is essential for vascular network stability, cell survival, and lamellipodial formation. J Cell Biol 172: 151-162, 2006.

20. Ettenson DS and Gotlieb AI: Endothelial wounds with disruption in cell migration repair primarily by cell proliferation. Microvasc Res 48: 328-337, 1994.

21. Eccles SA: Parallels in invasion and angiogenesis provide pivotal points for therapeutic intervention. Int J Dev Biol 48: 583-598, 2004.

22. Carmeliet P: Angiogenesis in health and disease. Nat Med 9: 653-660, 2003.

23. Fraley SI, Feng Y, Krishnamurthy R, Kim DH, Celedon A, Longmore GD and Wirtz D: A distinctive role for focal adhesion proteins in three-dimensional cell motility. Nat Cell Biol 12: 598-604, 2010.

24. van Nimwegen MJ and van de Water B: Focal adhesion kinase: A potential target in cancer therapy. Biochem Pharmacol 73: 597-609, 2007. 